\title{
Thermalization at RHIC
}

\author{
Ulrich Heinz \\ Physics Department, The Ohio State University, 174 West 18th Avenue, Columbus, OH 43210, USA
}

\begin{abstract}
Ideal hydroynamics provides an excellent description of all aspects of the single-particle spectra of all hadrons with transverse momenta below about $1.5-2 \mathrm{GeV} / c$ at RHIC. This is shown to require rapid local thermalization at a time scale below $1 \mathrm{fm} / c$ and at energy densities which exceed the critical value for color deconfinement by an order of magnitude. The only known thermalized state at such energy densities is the quark-gluon plasma (QGP). The rapid thermalization indicates that the QGP is a strongly interacting liquid rather than the weakly interacting gas of quarks and gluons that was previously expected.
\end{abstract}

\section{Collective flow as an indicator of thermalization}

Transverse flow in heavy-ion collisions is an unavoidable consequence of thermalization. Thermalization generates thermodynamic pressure in the matter created in the collision, which acts against the surrounding vacuum and causes rapid collective expansion ("flow") of the reaction zone. Since the quark-gluon plasma (QGP) is a thermalized system of deconfined quarks, antiquarks, and gluons, collective flow is a necessary result of QGP formation in heavy-ion collisions, and its absence could be taken as proof that no such plasma was ever formed. Its presence, however, does not automatically signal QGP formation. Detailed studies of the observed final state flow pattern are necessary to convince oneself that the reflected time-integrated pressure history of the collision region indeed requires a thermalized state in the early collision stage whose pressure and energy density are so high that it can no longer be mistaken as consisting of conventional hadronic matter.

Whereas radial flow (the azimuthally symmetric component of collective expansion transverse to the beam direction) integrates over the entire pressure history of the expanding fireball, anisotropic elliptic flow [1] (quantified by the second harmonic coefficient $v_{2}\left(y, p_{\perp} ; b\right)$ of a Fourier expansion in $\phi_{p}$ of the measured hadron spectrum $\left.d N /\left(d y p_{\perp} d p_{\perp} d \phi_{p}\right)[2]\right)$ is strongly weighted towards the very early stages of the expansion [3]. The higher the initial energy density, the less contributions it receives from the late, hadronic stage of the collision. At RHIC energies, elliptic flow almost saturates [4] before the energy density has dropped to the critical value $e_{\mathrm{cr}} \approx 1 \mathrm{GeV} / \mathrm{fm}^{3}$ $\left(T_{\text {cr }} \approx 170 \mathrm{MeV}\right)$ where normal hadrons can begin to form [5]. At higher LHC energies, the elliptic flow is expected to peak even before the onset of hadronization (see curve $c$ in Fig. 7 of [4]). At sufficiently high collision energies, elliptic flow is thus a QGP signature, probing the QGP equation of state $p(e)$. Its advantage over other "early signatures" is that it affects the bulk of the hadrons and can thus be measured differentially with high statistical accuracy. 
The reason why elliptic flow must develop early in the collision is easy to understand. Since individual nucleon-nucleon collisions produce azimuthally symmetric momentum spectra, any final state momentum anisotropies must be generated dynamically during the nuclear reaction. They require the existence of an initial spatial anisotropy of the reaction zone, either by colliding deformed nuclei such as $U+U$ [4, 6, 7], or by colliding spherical nuclei at non-zero impact parameter $b \neq 0$ (the practical method of choice so far). Final state interactions within the produced matter transfer the initial spatial anisotropy onto a final momentum anisotropy. Microscopic transport calculations [8, 9] show a monotonic dependence of $v_{2}$ on the opacity (density times scattering cross section) of the produced matter which is inversely related to its thermalization time. These studies strongly suggest that, for a given initial spatial anisotropy $\varepsilon_{x}$, the maximum momentum-space response $v_{2}$ is obtained in the ideal hydrodynamic limit which assumes perfect local thermal equilibrium at every space-time point (i.e. a thermalization time which is much shorter than any macroscopic time scale in the system). Any significant delay of thermalization (modelled, for example, as an initial free-streaming stage) causes a decrease of the initial spatial anisotropy without concurrent build-up of momentum anisotropies, thereby reducing the finally observed elliptic flow signal [4].

In this talk I will present results from hydrodynamic simulations of hadronic spectra and elliptic flow at RHIC energies. We will see that the hydrodynamic approach provides an excellent quantitative description of the bulk of the data and fails only for very peripheral $\mathrm{Au}+\mathrm{Au}$ collisions and/or at high $p_{\perp}>1.5-2 \mathrm{GeV} / c$. That the hydrodynamic approach fails if the initial nuclear overlap region becomes too small or the transverse momentum of the measured hadrons becomes too large is not unexpected. However, where exactly hydrodynamics begins to break down gives important information about the microscopic rescattering dynamics. What is really surprising is that the hydrodynamic approach works so well in central and semi-central collisions where it quantitatively reproduces the momenta of more than $99 \%$ of the particles. Below $p_{\perp}=1.5 \mathrm{GeV} / c$ the elliptic flow data [11, 12, 13] actually exhaust the hydrodynamically predicted [4, 14, 15, 16, 17] upper limit. The significance of this agreement can hardly be overstressed, since it implies one of the biggest surprises so far at RHIC: the produced matter (which I call QGP since this is only known viable concept of thermalized matter at $\left.e \sim(10-20) e_{\mathrm{cr}}\right)$ is not the originally expected weakly interacting gas of quarks and gluons ( $w \boldsymbol{Q G P}$ for "weakly interacting quark-gluon plasma"), but a strongly coupled liquid with extremely small viscosity ( $\boldsymbol{Q} \boldsymbol{Q} \boldsymbol{G P}$ for "strongly interacting quarkgluon plasma" [18]). In fact, upper limits [19] on the dimensionless ratio of viscosity to entropy density, $\eta / s$, based on an analysis of the RHIC elliptic flow data, indicate that the sQGP is less viscous, by about an order of magnitude, than even liquid helium below the transition to superfluidity. The $S Q G P$ is therefore the most ideal fluid ever observed!

\section{Hydrodynamic expansion in heavy-ion collisions}

The natural language for describing collective flow phenomena is hydrodynamics. Its equations control the space-time evolution of the pressure, energy and particle densities and of the local fluid velocity. The system of hydrodynamic equations is closed by 
specifying an equation of state which gives the pressure as a function of the energy and particle densities. In the ideal fluid (non-viscous) limit, the approach assumes that the microscopic momentum distribution is thermal at every point in space and time (note that this does not require chemical equilibrium - chemically non-equilibrated situations can be treated by introducing into the equation of state non-equilibrium chemical potentials for each particle species [20, 21, 22]). Small deviations from local thermal equilibrium can in principle be dealt with by including viscosity, heat conduction and diffusion effects, but such a program is made difficult in practice by a number of technical and conceptual questions [23] and has so far not been successfully applied to relativistic fluids. Stronger deviations from local thermal equilibrium require a microscopic phasespace approach (kinetic transport theory), but in this case the concepts of equation of state and local fluid velocity field themselves become ambiguous, and the direct connection between flow observables and the equation of state of the expanding matter is lost.

The assumption of local thermal equilibrium in hydrodynamics is an external input, and hydrodynamics offers no direct insights about the equilibration mechanisms. It is clearly invalid during the initial particle production and early recattering stage, and it again breaks down towards the end when the matter has become so dilute that rescattering ceases and the hadrons "freeze out". The hydrodynamic approach thus requires a set of initial conditions for the dynamic variables at the earliest time at which the assumption of local thermal equilibrium is applicable, and a "freeze-out prescription" at the end. For the latter we use the Cooper-Frye algorithm [24] which implements an idealized sudden transition from perfect local thermal equilibrium to free-streaming. This is not unreasonable because freeze-out (of particle species $i$ ) is controlled by a competition between the local expansion rate $\partial \cdot u(x)$ (where $u^{\mu}(x)$ is the fluid velocity field) and the local scattering rate $\sum_{j}\left\langle\sigma_{i j} v_{i j}\right\rangle \rho_{j}(x)$ (where the sum goes over all particle species with densities $\rho_{j}(x)$ and $\left\langle\sigma_{i j} v_{i j}\right\rangle$ is the momentum-averaged transport cross section for scattering between particle species $i$ and $j$, weighted with their relative velocity); while the local expansion rate turns out to have a rather weak time-dependence, the scattering rate drops very steeply as a function of time, due to the rapid dilution of the particle densities $\rho_{j}$ [25], causing a rapid transition to free-streaming. - A better algorithm [26, 14] switches from a hydrodynamic description to a microscopic hadron cascade at or shortly after the quark-hadron transition, before the matter becomes too dilute, and lets the cascade handle the freeze-out kinetics. This also correctly reproduces the final chemical composition of the fireball, since the particle abundances already freeze out at hadronization, due to a lack of particle-number changing inelastic rescattering processes in the hadronic phase. The resulting radial flow patterns [14] from such an improved freeze-out algorithm don't differ much from our simpler Cooper-Frye based approach.

\section{Hydrodynamic radial flow and RHIC particle spectra}

We have solved the relativistic equations for ideal hydrodynamics, as described in [4]. To simplify the numerical task, we imposed boost-invariant longitudinal expansion analytically [1, 27]. As long as we focus on the transverse expansion dynamics near 
midrapidity (the region which most RHIC experiments cover best), this does not give up any essential physics.

We use an EOS which is constructed by matching a free ideal quark-gluon gas above $T_{\mathrm{cr}}$ to a realistic hadron resonance gas [28, 29] below $T_{\mathrm{cr}}$, using a Maxwell construction and fixing $T_{\mathrm{cr}}=165 \mathrm{MeV}$ to reproduce lattice QCD results [5]. The Maxwell construction leads to an artificial first order transition, with a latent heat of $1.15 \mathrm{GeV} / \mathrm{fm}^{3}$, whereas the lattice QCD data indicate either a very weak first order transition or a rapid, but smooth, crossover. As long as this crossover is very steep (as the lattice data indicate [5]), the dynamical consequences of our first-order idealization of the EOS are not expected to be significant.

We have used two variants of the hadron resonance gas below $T_{\mathrm{cr}}$ : In our first sets of calculations 1999-2002, we assumed the hadron resonance gas to be in chemical equilibrium until kinetic freeze-out. RHIC data on particle abundance ratios show, however, that hadronic particle yields freeze out directly at $T_{\mathrm{cr}}$ [30], due to inefficiency of particlechanging inelastic reactions in the relatively dilute and rapidly expanding hadron gas phase below $T_{\mathrm{cr}}$. More recent hydrodynamic calculations [21, 31] therefore include nonequilibrium chemical potentials [20, 21, 22] ensuring number conservation for individual (stable) hadron species below $T_{\mathrm{cr}}$. This does not affect the equation of state $p(e)$ and therefore leads to the same flow pattern as the chemical equilibrium EOS [21]. What does change, however, is the relationship between energy density $e$ and temperature $T$ since in the chemical non-equilibrium case more of the energy is stored in the rest masses of heavy baryons and antibaryons (which are not allowed to annihilate as the temperature drops). The same decoupling energy density $e_{\mathrm{dec}}=0.075 \mathrm{GeV} / \mathrm{fm}^{3}$ obtained from a fit to RHIC data (see next paragraph) therefore corresponds to $T_{\mathrm{dec}}=130 \mathrm{MeV}$ in the chemical equilibrium case, but drops to the much lower value $T_{\mathrm{dec}}=100 \mathrm{MeV}$ if hadronic chemical freeze-out at $T_{\mathrm{cr}}=165 \mathrm{MeV}$ is properly taken into account [21, 31].

The initial and final conditions for the hydrodynamic evolution are fixed by fitting the total charged multiplicity $d N_{\mathrm{ch}} / d y$ and the pion and proton spectra at midrapidity in central $(b=0)$ collisions. (All calculated hadron spectra include feeddown from decays of unstable hadron resonances [32].) Since these two hadrons have quite different masses, their spectra allow for the independent extraction of the temperature and average radial flow velocity at freeze-out [33]. The freeze-out temperature together with the total charged multiplicity determines the total fireball entropy which in ideal hydrodynamics is preserved during the expansion. During the early, predominantly longitudinal expansion stage, where the fireball volume increases linearly with proper time $\tau$, this constraint fixes the product $\tau \cdot \int d^{2} r_{\perp} s\left(\boldsymbol{r}_{\perp}, \tau\right)$ where $s\left(\boldsymbol{r}_{\perp}, \tau\right)$ is the entropy density distribution in the plane transverse to the beam. Since the initial shape of this distribution is fixed by the nuclear overlap geometry, using a Glauber model ansatz [17], this constraint determines the normalization of $s\left(\boldsymbol{r}_{\perp}, \tau_{\text {eq }}\right)$ (i.e. the central entropy density $s_{0}=s\left(\mathbf{0}, \tau_{\mathrm{eq}}\right)$ ) as a function of the initial thermalization time $\tau_{\text {eq. }}$. The Glauber model contains one additional parameter: the ratio of "soft" and "hard" components of initial particle production. Since these scale with the transverse density of wounded nucleons and binary nucleon-nucleon collisions, respectively, their ratio can be determined from the collision centrality dependence of the produced charged multiplicity $d N_{\mathrm{ch}} / d y$ [17]. We use $25 \%$ hard and $75 \%$ soft contributions to the initial entropy production [34]. The initial thermalization time $\tau_{\text {eq }}$, finally, is fixed by the need for the hydrodynamic evolution to have 
enough time to generate the finally observed radial flow. At RHIC energies, we find that this requires thermalization times $\tau_{\mathrm{eq}} \leq 0.6 \mathrm{fm} / c$. In our calculations we take the upper limit of this interval. In Au+Au collisions at $\sqrt{s}=100 \mathrm{~A} \mathrm{GeV}$, the initial central entropy density at this time is $s_{0}=110 \mathrm{fm}^{-3}$, corresponding to an initial central energy density ${ }^{1}$ $e_{0}=30 \mathrm{GeV} / \mathrm{fm}^{3} \approx 30 e_{\mathrm{cr}}$ and an initial central temperature $T_{0}=360 \mathrm{MeV} \approx 2 T_{\mathrm{cr} .}{ }^{2}$
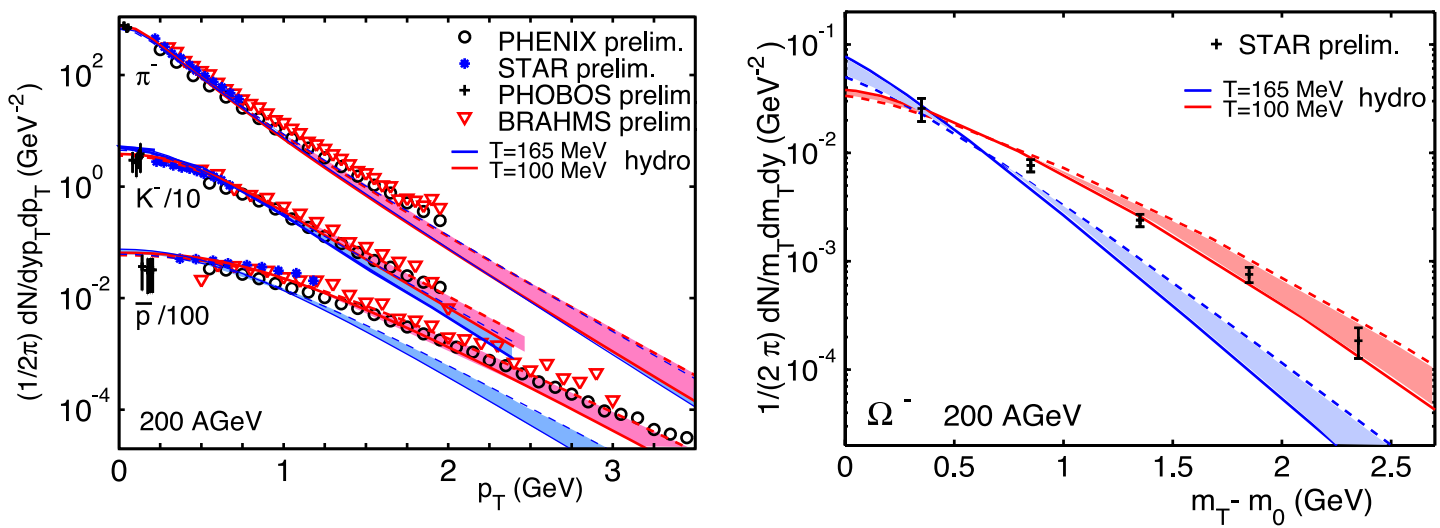

FIGURE 1. (color online) Negative pion, kaon, antiproton, and $\Omega$ spectra from central Au+Au collisions at $\sqrt{s}=200 \mathrm{~A} \mathrm{GeV}$, as measured by the four RHIC experiments [35, 36, 37, 38, 39]. The curves show hydrodynamical calculations as described in the text.

In Fig. 11 show the (absolutely normalized) single particle $p_{\perp}$-spectra for negatively charged pions, kaons and antiprotons (left panel) as well as $\Omega$ baryons (right panel) measured in $\mathrm{Au}+\mathrm{Au}$ collisions at RHIC together with hydrodynamical results [31]. In order to illustrate the effect of additional radial flow generated by elastic scattering in the late hadronic stage below $T_{\mathrm{cr}}$, two sets of curves are shown: the lower (blue) bands correspond to kinetic decoupling at $T_{\mathrm{cr}}=165 \mathrm{MeV}$, whereas the upper (red) bands assume decoupling at $T_{\mathrm{dec}}=100 \mathrm{MeV}$. The width of the bands indicates the sensitivity of the calculated spectra to an initial transverse flow of the fireball already at the time of thermalization: The lower end assumes no initial transverse flow whereas the upper end implements an initial radial flow profile $v_{r}\left(r_{\perp}, \tau_{\mathrm{eq}}\right)=\tanh \left(\alpha r_{\perp}\right)$ with $\alpha=0.02 \mathrm{fm}^{-1}$ at $\tau_{\text {eq }}=0.6 \mathrm{fm} / c$ (which seems to be slightly preferred by the data). The hydrodynamic model output shows [4] that it takes about $9-10 \mathrm{fm} / c$ until the fireball has become sufficiently dilute to completely convert to hadronic matter and another $7-8 \mathrm{fm} / c$ to completely decouple. Figure 1 shows clearly that by the time of hadronization hydrodynamics has not yet generated enough radial flow to reproduce the measured proton and $\Omega$ spectra; these heavy hadrons, which are particularly sensitive to radial flow effects, require the additional collective "push" created by resonant (quasi)elastic interactions during the fairly long-lived hadronic rescattering stage between $T_{\mathrm{cr}}$ and $T_{\mathrm{dec}}$. Even though independent flow fits to $\pi, K, p$ spectra on the one hand and multistrange hyperon spectra on the other [40] suggest (within some systematic uncertainty related to the strong

\footnotetext{
${ }^{1}$ When averaged over the transverse profile, this corresponds to $\langle e\rangle=13 \mathrm{GeV} / \mathrm{fm}^{3}$ which is still a good order of magnitude above the critical value for deconfinement, $e_{\mathrm{cr}}=0.6-1 \mathrm{GeV} / \mathrm{fm}^{3}[5]$.

${ }^{2}$ If the initial QGP is not chemically equilibrated, but rather dominated by gluons, this initial temperature could be as high as $460 \mathrm{MeV}$.
} 
anticorrelation between the extracted flow and temperature values) that $\Xi$ and $\Omega$ hyperons decouple slightly earlier (at somewhat higher temperature and with less radial flow) than pions, kaons, and protons, their immediate decoupling directly at hadronization is obviously not dynamically consistent with the successful hydrodynamic approach.

The strong flattening of the (anti)proton spectra by radial flow provides a natural explanation for the (initially puzzling) experimental observation that for $p_{\perp}>2 \mathrm{GeV} / c$ antiprotons become more abundant than pions. For a hydrodynamically expanding thermalized fireball, at relativistic transverse momenta $p_{\perp} \gg m_{0}$ all hadron spectra have the same slope [33], and at fixed $m_{\perp} \gg m_{0}$ their relative normalization is given by $\left(g_{i} \lambda_{i}\right) /\left(g_{j} \lambda_{j}\right)$ (where $g_{i, j}$ is the spin-isospin degeneracy factor and $\lambda_{i, j}=e^{\mu_{i, j} / T}$ is the fugacity of hadron species $i, j$ ). Due to the large antiproton chemical potential at kinetic freeze-out which is required to maintain the $\bar{p}$ abundance at temperatures below chemical freeze-out, this asymptotic $\bar{p} / \pi^{-}$ratio is predicted to be about 28 [41]. Within the hydrodynamic approach the surprising fact is therefore not that $\bar{p} / \pi>1$ at $p_{\perp}>2 \mathrm{GeV} / c$, but that this ratio seems to saturate around 1 and never much exceeds this value [35]. As we will see below, this is a signature of the beginning breakdown of the hydrodynamic model which stops working for baryons with transverse momenta above about $2.5 \mathrm{GeV} / c$.

As shown elsewhere (see Fig. 1 in [42]), once the hydrodynamic model parameters have been fixed to describe pions, protons, and total multiplicity in central $\mathrm{Au}+\mathrm{Au}$ collisions, the model describes these and all other hadron spectra not only in central, but also in peripheral collisions, up to impact parameters of about $10 \mathrm{fm}$ and with similar quality. No additional parameters enter at non-zero impact parameter - only the initial conditions change due to the changing overlap geometry, but this is completely accounted for by the Glauber model. Therefore, hydrodynamic results for the elliptic flow, discussed in the next Section, are parameter-free predictions of the model.

\section{Hydrodynamic elliptic flow and RHIC data}

Figure 2 shows the predictions for the elliptic flow coefficient $v_{2}$ from $\mathrm{Au}+\mathrm{Au}$ collisions at RHIC, together with the data [11, 12, 43]. For impact parameters $b \leq 7 \mathrm{fm}$ (corresponding to $n_{\mathrm{ch}} / n_{\max } \geq 0.5$ ) and transverse momenta $p_{\perp} \leq 1.5-2 \mathrm{GeV} / c$ the data are seen to exhaust the upper limit for $v_{2}$ obtained from the hydrodynamic calculations. For larger impact parameters $b>7 \mathrm{fm}$ the $p_{\perp}$-averaged elliptic flow $v_{2}$ increasingly lags behind the hydrodynamic prediction. It is tempting to attribute this to a lack of early thermalization when the initial overlap region becomes too small [34]. However, the work by Teaney [14], who coupled hydrodynamic evolution of the quark-gluon plasma above $T_{\mathrm{cr}}$ with a microscopic kinetic evolution of a hadronic resonance gas using RQMD below $T_{\text {cr }}$, showed that at least a strong contributing factor to the lack of elliptic flow in peripheral collisions (as well as in central collisions at lower energy) is the large viscosity in the late hadronic stage. If the initial energy density is not high enough, the initial spatial deformation does not completely disappear until hadronization. Whereas ideal hydrodynamics then predicts a continued growth of the elliptic flow during the hadronic stage, driven by the still existing anisotropies in the pressure gradients, realistic hadron 

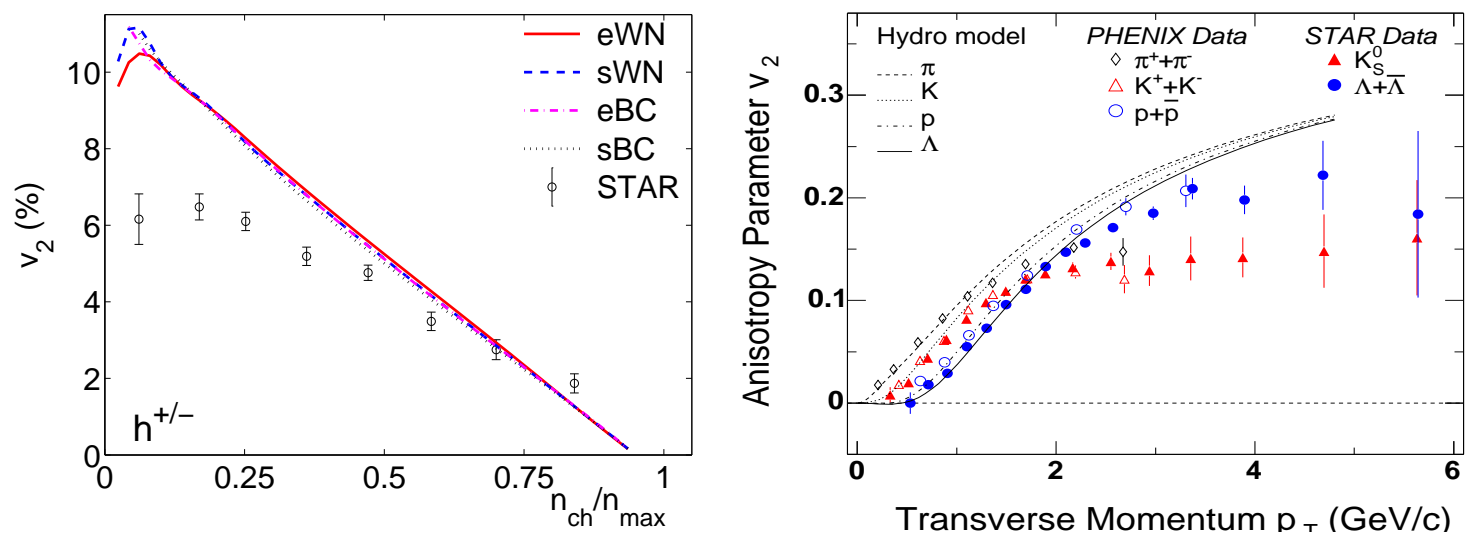

FIGURE 2. (color online) Elliptic flow coefficient $v_{2}$ for all charged particles from $130 \mathrm{~A} \mathrm{GeV} \mathrm{Au+Au}$ collisions (left panel [17]) and for several identified hadron species from $200 \mathrm{~A} \mathrm{GeV} \mathrm{Au+Au} \mathrm{collisions}$ (right panel [44]), compared with hydrodynamic predictions. The left panel shows the $p_{\perp}$-averaged elliptic flow as a function of collision centrality, parametrized by the charged multiplicity density $n_{\mathrm{ch}}$ at midrapidity ( $n_{\max }$ corresponds to the largest value in central collisions). The right panel shows the differential elliptic flow $v_{2}\left(p_{\perp}\right)$ for minimum bias collisions. The data were collected by STAR and PHENIX [11, 12, 43]. The curves in the left panel are hydrodynamic calculations corresponding to different choices for the initial energy density profile (see [17] for details). The curves in the right panel were first published in [16].

cascades produce very little additional elliptic flow, indicating that the hadronic matter is very viscous. To completely saturate the elliptic flow value predicted by ideal hydrodynamics thus requires not only very rapid thermalization at the beginning of the reaction, but also a sufficiently long lifetime of the thermalized QGP stage such that the initial spatial anisotropy has time to fully decay and the momentum anisotropy can fully develop before the system enters the viscous hadron gas phase. This explains why the elliptic flow lags behind the hydrodynamic prediction at RHIC in peripheral $\mathrm{Au}+\mathrm{Au}$ collisions at midrapidity, in minimum bias $\mathrm{Au}+\mathrm{Au}$ collisions at forward and backward rapidities $[21,45,46]$, as well as quite generally at lower collision energies [47, 48]. From an analysis of all the available data we recently concluded [46] that an average initial energy density $\langle e\rangle_{\tau_{\mathrm{eq}}} \geq 10 \mathrm{GeV} / \mathrm{fm}^{3}$ appears to be necessary to provide sufficient evolution time in the plasma phase for full development of the momentum anisotropy before hadronization and therefore full saturation of the hydrodynamic elliptic flow limit.

The $p_{\perp}$-dependence of $v_{2}$ (right panel of Fig. 2) tells another story: whereas the hydrodynamic model predicts a continuous rise of $v_{2}$ with increasing $p_{\perp}$, the measured elliptic flow appears to saturate at high $p_{\perp}$. Baryon elliptic flow (here shown are protons and $\Lambda$ hyperons, but $\Xi$ and $\Omega$ hyperons follow the same systematics [49]) saturates at a higher value than meson (pion and kaon) elliptic flow. These saturation patterns imply a break of the data away from the hydrodynamic prediction, and again this break happens at higher $p_{\perp}$ for baryons $\left(p_{\perp}^{\text {break }} \simeq 2.3-2.5 \mathrm{GeV} / c\right)$ than for mesons $\left(p_{\perp}^{\text {break }} \simeq 1.5 \mathrm{GeV} / c\right)$. Below these break points, the elliptic flow of all hadrons measured so far is very well described by hydrodynamics. In particular the hydrodynamically predicted mass splitting of $v_{2}$ at low $p_{\perp}$ is perfectly reproduced by the data. Hydrodynamics thus gives an excellent description of all hadron spectra below $p_{\perp}=1.5 \mathrm{GeV} / c$. Due to the 
exponentially falling $p_{\perp}$ spectra, this includes more than $99 \%$ of all produced hadrons, so that it is fair to say that the bulk of the fireball matter formed in Au+Au collisions at RHIC behaves like a perfect fluid.

In [16] we showed that the slope of $v_{2}\left(p_{\perp}\right)$ and its mass splitting are sensitive to the equation of state. The mass splitting is larger for an EOS which includes a quarkhadron phase transition than for a pure resonance gas EOS extrapolated to arbitrarily high temperatures. Although requiring many more systematic model studies than so far available, these features offer the perspective of extracting detailed knowledge on the EOS during the early expansion stages from combined radial and elliptic flow measurements. So far we only know qualitatively (see Fig. 2 in [42] and the discussion in [14]) that, for given angle-averaged $p_{\perp}$ spectra, the measured elliptic flow prefers an EOS that includes a phase transition at $T_{\mathrm{cr}}=165 \mathrm{MeV}$ over one without such a transition.

The excellent agreement between data and hydrodynamic model at transverse momenta below about $2 \mathrm{GeV}$ becomes even more impressive after one begins to realize how easily it is destroyed: Parton cascade simulations with standard HIJING input generate almost no elliptic flow and require an artificial increase of the opacity of the partonic matter by a factor 80 to reproduce the RHIC data [9]. This shows that the QGP is much more strongly coupled than achievable within a standard perturbative QCD approach. Hadronic cascades of the RQMD and URQMD type (in which the high-density initial state is parametrized by non-interacting, pressureless QCD strings) predict [50] too little elliptic flow and a decrease of $v_{2}$ from SPS to RHIC, contrary to the data. Generically, models that don't include very strong rescattering in the very early fireball expansion stage fail to reproduce the elliptic flow data.

As I will show in the next section, any significant delay of thermalization immediately leads to a significant loss of elliptic flow signal - a fact that can be used to establish tight upper limits on the thermalization time scale. Even partial thermalization doesn't work: In [51] we studied the transverse hydrodynamic evolution of a toy model in which only transverse momenta were thermalized while in longitudinal direction the fireball evolved ballistically. (One can think of this as implementing into the transverse hydrodynamics a very large value for the shear viscosity.) Since in this model there is no longitudinal pressure, more work is done by the pressure in the transverse direction, and one has a stiffer effective equation of state $p=e / 2$ (instead of the ideal quark-gluon gas $\operatorname{EOS} p=e / 3)$ driving the transverse expansion. To obtain the same $p_{\perp}$-spectra as before one must thus retune the initial conditions and let the hydrodynamic expansion start later, in order to avoid getting too much radial flow. The consequence of this is a reduction of the elliptic flow $v_{2}$ by almost a factor 2 , to levels far below the experimental data. This exercise demonstrates a strong sensitivity of the elliptic flow to early shear viscosity. A complementary analysis by Teaney, who discussed late-stage viscous effects on hadron spectra, elliptic flow and HBT radii in [19], arrives at similar conclusions. This sensitivity of $v_{2}$ to shear viscosity opens the perspective of limiting the shear viscosity of a quark-gluon plasma experimentally by performing systematic comparisons between elliptic flow data and viscous (i.e. non-ideal) hydrodynamics. This has recently led to renewed interest in practical implementations of viscous relativistic fluid dynamics [52]. 


\section{Upper limits for the QGP thermalization time}

The agreement between the elliptic flow data at RHIC and the hydrodynamic simulations can be used to set an upper limit on the thermalization time scale in the dense partonic matter formed in the collision which is independent from the value $\tau_{\text {eq }} \leq 0.6 \mathrm{fm} / c$ obtained two chapters earlier by fitting the model to central collision spectra. Let me illustrate this by referring to Fig. 3 which, to some extent, is a different way of plotting the results shown in the left panel of Fig. 2. In ideal hydrodynamics, the final elliptic flow $v_{2}$ is directly proportional to the initial spatial deformation $\varepsilon_{x}$, with the propor-

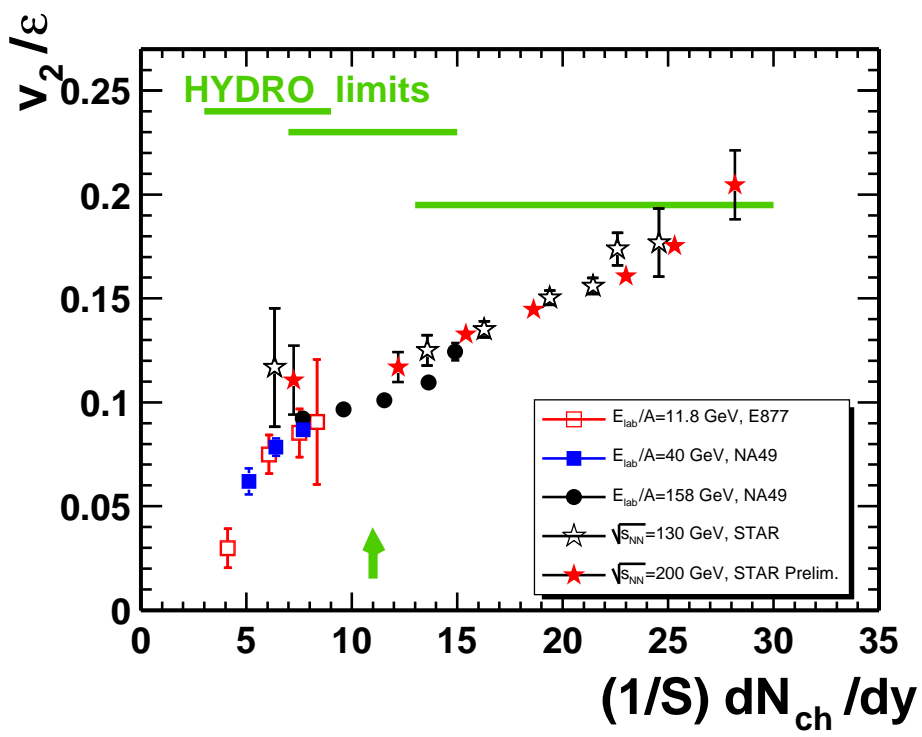

FIGURE 3. (color online) Scaled elliptic flow $v_{2} / \varepsilon_{x}$ as a function of $(1 / S) d N_{\mathrm{ch}} / d y$ (i.e. the charged multiplicity density per unit transverse overlap area $S$ ), for $\mathrm{Pb}+\mathrm{Pb}$ and $\mathrm{Au}+\mathrm{Au}$ collisions of different centralities at various collision energies, as compiled by the NA49 Collaboration [47]. The lines indicate the hydrodynamically predicted values at RHIC, SPS and AGS energies [4] (see text for details).

tionality constant being controlled by the equation of state through the speed of sound $c_{s}^{2}=\partial p / \partial e[1]$. The reason for this scaling is that the hydrodynamic equations of motion are scale-free, and that therefore the initial condition for $\varepsilon_{x}$ sets the scale for the final momentum anisotropy. This scaling assumes that the momentum anisotropy can fully develop, i.e. that the hydrodynamic evolution is not cut short by decoupling before the spatial anisotropy $\varepsilon_{x}$ has completely disappeared. As discussed in the previous chapter, this assumption is justified near midrapidity in central and near-central $\mathrm{Au}+\mathrm{Au}$ collisions at RHIC, but breaks down more and more severely as one goes to more peripheral collisions, away from midrapidity, or to lower collision energies. This is at least part of the explanation of the difference between the data points in Fig. 3 and the horizontal lines labelled "HYDRO limits" [14].

These horizontal lines represent the fully developed hydrodynamic elliptic flow (scaled by the initial deformation $\varepsilon_{x}$ ) for minimum bias $\mathrm{Au}+\mathrm{Au}$ or $\mathrm{Pb}+\mathrm{Pb}$ collisions for three different effective sound speeds, reflecting the effective stiffness of our hadron gas + quark-gluon plasma EOS for collisions at (from right to left or bottom to top) 
RHIC, SPS and AGS energies. They were extracted from the elliptic flow excitation function for $b=7 \mathrm{fm} \mathrm{Pb}+\mathrm{Pb}$ collisions given in Fig. 14 of [4], noting that $b=7 \mathrm{fm}$ is roughly equal to the average impact parameter in minimum bias $\mathrm{Au}+\mathrm{Au}$ collisions. The RHIC value for $v_{2} / \varepsilon_{x}$ is lower than the ones for SPS and AGS energies since RHIC collisions spend more of the relevant time when the elliptic flow builds up in the soft phase transition region, compared to SPS and AGS where most or all of that time is spent in the stiffer hadron gas phase [4]. The increasing discrepancy between these lines and the data as one decreases the collision energy indicates the inability of the hadron gas phase (which gains more and more dynamical weight) to respond to the spatial deformation of the reaction zone. This is a late-stage effect and does not necessarily indicate a lack of early thermalization.

However, some lack of early thermalization may still contribute to this discrepancy. Let us, for the sake of the argument, concentrate on Au+Au collisions at $\sqrt{s}=200 \mathrm{~A} \mathrm{GeV}$ (solid red stars in Figure 3) and pretend that, in fact, the entire discrepancy between data and theory is due to inefficient thermalization at the beginning of the collision. Let us model this inefficiency in a somewhat black-and-white fashion, by assuming that the system evolves by free-streaming (i.e. without any interactions at all) for a time $\Delta \tau$, after which it instantaneously thermalizes and continues to evolve according to the laws of ideal fluid dynamics. As shown in [4] (for a slightly more general argument see [41]), free-streaming results in a radial growth of the source which reduces its initial spatial deformation $\varepsilon_{x}$ according to

$$
\frac{\varepsilon_{x}\left(\tau_{0}+\Delta \tau\right)}{\varepsilon_{x}\left(\tau_{0}\right)}=\left[1+\frac{(c \Delta \tau)^{2}}{\left\langle\boldsymbol{r}_{\perp}^{2}\right\rangle_{\tau_{0}}}\right]^{-1} .
$$

Due to the hydrodynamic scaling of the final $v_{2}$ with the initial spatial deformation $\varepsilon_{x}$, the final elliptic flow will be reduced by at least the same factor (and by even more if, after this delay, the hydrodynamic stage is no longer sufficiently long-lived to fully develop the elliptic flow). By assigning all of the "missing" elliptic flow in Figure 3 to this "delayed thermalization" effect, we can therefore extract a conservative upper limit for the thermalization time scale $\Delta \tau$.

Note that even if (contrary to the above arguments based on Teaney's work [14]) our assumption were true that all of the "missing" elliptic flow in Figure 3 were due to inefficient thermalization in the initial partonic stage (rather than in the late hadronic stage), this procedure will still only yield an upper limit for the thermalization time $\Delta \tau$ : In real life, thermalization doesn't happen suddenly after a period of no scattering at all, but gradually. Any type of scattering, however, will generate anisotropic evolution (albeit not as strongly anisotropic as in ideal fluid dynamics), i.e. faster expansion into the reaction plane than perpendicular to it, thereby reducing the spatial deformation $\varepsilon_{x}$ (and thus the final $v_{2}$ ) faster than given by Eq. (1).

Let us now use this to extract an upper limit for the thermalization time $\Delta \tau$ in $b \approx 7 \mathrm{fm}$ $\mathrm{Au}+\mathrm{Au}$ collisions (4th solid red star from the right in Fig. 3). According to the Figure, for this impact parameter $v_{2} / \varepsilon_{x}$ is about $75 \%$ of the hydrodynamic value. Equating this with $\varepsilon_{x}\left(\tau_{0}+\Delta \tau\right) / \varepsilon_{x}\left(\tau_{0}\right)$, and inserting $\left\langle r_{\perp}^{2}\right\rangle_{\tau_{0}}^{1 / 2}=3.5 \mathrm{fm}$ for $b \approx 7 \mathrm{fm} \mathrm{Au}+\mathrm{Au}$, we find $\Delta \tau \approx 2 \mathrm{fm} / \mathrm{c}$ for this upper limit. For second solid red star from the right, the experimental value corresponds to $90 \%$ of the hydrodynamic limit, and the resulting upper limit for 
the thermalization time is $\Delta \tau \approx 1 \mathrm{fm} / c$. The right-most point in Figure 3 allows for a nonzero thermalization time only by virtue of its large error bar (and, of course, systematic uncertainties in the exact vertical position of the theoretical horizontal line).

We see that, even if we assign all of the "missing" elliptic flow to inefficient early thermalization, the resulting window for the thermalization time scale is narrow and not larger than about $2 \mathrm{fm} / c$ in minimum bias $\mathrm{Au}+\mathrm{A}$ collisions and certainly less than $1 \mathrm{fm} / c$ in central $\mathrm{Au}+\mathrm{Au}$ collisions. This window becomes even narrower when accounting for the well-documented [14] inefficiency of the late hadronic stage to generate elliptic flow. When taken together, these arguments yield very tight limits on the thermalization time scale in the dense matter formed early in the collision which, I think, are fairly represented (and not underestimated) by the value $\tau_{\mathrm{eq}}=0.6 \mathrm{fm} / c$ used as starting time in our hydrodynamic simulation. (Let me remind you once again that this time was determined in a different way, using only information from central collisions, and that it therefore enters my network of arguments for early thermalization as an independent "data point".)

It is, of course, unsatisfactory to see in Figure 3 the ideal hydrodynamic limit just being reached at one point, namely in the most central $\mathrm{Au}+\mathrm{Au}$ collisions at the highest available collision energy. The natural reaction of any serious physicist should be that this single point coincidence must be accidental, and that the Figure really shows incompatible tendencies in theory and data. The only problem with this "conservative" reaction is that it should be impossible to create more elliptic flow than predicted by ideal fluid dynamics! If at larger values for $(1 / S) d N_{\mathrm{ch}} / d y$ the data for $v_{2} / \varepsilon_{x}$ indeed continued to grow significantly above the hydrodynamic limit (as suggested by the tendency of the data in Figure 3), this would create very serious problems for theory. It is therefore very important to try to extend the range of $(1 / S) d N_{\mathrm{ch}} / d y$ to larger values, by studying either semi-central $\mathrm{Pb}+\mathrm{Pb}$ collisions at the LHC or central $\mathrm{U}+\mathrm{U}$ collisions at RHIC [4, 46]. In order to support our present understanding of heavy-ion collision dynamics it will be essential to confirm that for $(1 / S) d N_{\mathrm{ch}} / d y>30$ the ratio $v_{2} / \varepsilon_{x}$ saturates at the hydrodynamic limit, showing only a very weak growth between RHIC and LHC (resulting from a stiffening of the effective equation of state) [4].

\section{Hadronization via quark coalescence - evidence for deconfinement}

We already mentioned that the right panel of Figure 2 indicates a characteristic difference between mesons and baryons in the way their elliptic flow breaks away at intermediate $p_{\perp}$ from the hydrodynamic behaviour at low $p_{\perp}$. In the same intermediate $p_{\perp}$ region a similar meson-baryon split is seen in the nuclear enhancement factors, specifically the ratio $R_{C P}$ between the various hadron yields (scaled by the number of binary nucleon-nucleon collisions) measured in central and peripheral $\mathrm{Au}+\mathrm{Au}$ collisions [53]. It is quite striking that in this ratio the $\phi$ meson data fall together with the much lighter kaons [53] and not with the almost equally heavy protons [54] which instead follow the other measured baryons $\Lambda, \Xi$, and $\Omega$ [53, 54]. This systematics indicates that hadron production at intermediate transverse momenta, $1.5 \mathrm{GeV} / c<p_{\perp}<5-6 \mathrm{GeV} / c$, is controlled by the number of valence quarks inside the hadron rather than by its mass. 
Mass scaling, as seen for the elliptic flow at low $p_{\perp} \leq 1.5 \mathrm{GeV} / c$ (see Fig. 2 right panel), is characteristic of thermalization and hydrodynamic flow; a scaling with the number of valence quarks, on the other hand, is not consistent with hydrodynamics and rather suggests a quark coalescense picture [55]. In fact, in the transition region around $1.5-$ $2.5 \mathrm{GeV} / c$ where hydrodynamics begins to break down, a quark coalescence approach works extremely well, as I will now show.

The coalescence model was previously developed for and applied to the formation of deuterons and other light nuclei from nucleons in low- and high-energy nuclear collisions (see [56, 57] and references therein). The formalism developed there is most useful if the wave function of the cluster formed by the coalescence process has a narrow internal momentum distribution (i.e. it is relatively large). Clearly, hadrons have a much wider internal momentum distribution than deuterons, but if the total hadron momentum is sufficiently large (here we are interested in momenta $>1.5 \mathrm{GeV} / c$ ), neglecting the internal relative momenta of the quarks inside the hadron may still be a reasonable approximation. This is how the coalescence model has been applied to quark coalescence at RHIC by most authors [58, 59, 60] (for exceptions see [61, 62]).

One starts from the following expressions for the invariant momentum spectrum for mesons $M$ and baryons $B$ [56, 57]:

$$
\begin{aligned}
E \frac{d N_{M}}{d^{3} p}= & \sum_{\alpha \bar{\beta}} \int \frac{p_{\mu} d \boldsymbol{\sigma}^{\mu}(x)}{(2 \pi)^{3}} \int d^{3} q\left|\psi_{\boldsymbol{p}}^{M, \alpha \beta}(\boldsymbol{q})\right|^{2} f_{\alpha}\left(\boldsymbol{p}+\frac{1}{2} \boldsymbol{q}, x\right) f_{\bar{\beta}}\left(\boldsymbol{p}-\frac{1}{2} \boldsymbol{q}, x\right) \\
E \frac{d N_{B}}{d^{3} p}= & \sum_{\alpha \beta \gamma} \int \frac{p_{\mu} d \boldsymbol{\sigma}^{\mu}(x)}{(2 \pi)^{3}} \int d^{3} q_{1} d^{3} q_{2}\left|\psi_{\boldsymbol{p}}^{B, \alpha \beta \gamma}\left(\boldsymbol{q}_{1}, \boldsymbol{q}_{2}\right)\right|^{2} \\
& \times f_{\alpha}\left(\boldsymbol{p}+\frac{1}{2} q_{1}, x\right) f_{\beta}\left(\boldsymbol{p}+\frac{1}{2} \boldsymbol{q}_{2}, x\right) f_{\gamma}\left(\boldsymbol{p}-\frac{1}{2}\left(\boldsymbol{q}_{1}+\boldsymbol{q}_{2}\right), x\right)
\end{aligned}
$$

The $\psi$ 's are the internal wavefunctions of the hadrons, expressed as functions of the relative momenta of their valence quarks, and the $f$ 's are the valence quark phase-space distributions. The sums extend over all spin, isospin and color channels contributing to the desired hadronic final state. The outer integral goes over the hadronization surface $\sigma(x)$. In the narrow wave function limit $\boldsymbol{q}_{i}=0$, and under certain assumptions about $\sigma(x)$ (e.g. that its spatial anisotropy is small so that the momentum anisotropy of the spectra is dominated by the momentum anisotropy of the quark distribution functions $f_{\alpha, \beta, \gamma}(\boldsymbol{p}, x)$ ), these formulae imply that the azimuthal distributions of the hadrons are related to those of the quarks by

$$
\frac{d N_{M}}{d \phi_{p}} \sim\left(\frac{d N_{q}}{d \phi_{p}}\right)^{2}, \quad \frac{d N_{B}}{d \phi_{p}} \sim\left(\frac{d N_{q}}{d \phi_{p}}\right)^{3},
$$

and that the elliptic flow coefficients (neglecting small nonlinear terms) satisfy [60]

$$
v_{2}^{M}\left(p_{\perp}\right) \approx v_{2}^{\alpha}\left(\frac{p_{\perp}}{2}\right)+v_{2}^{\bar{\beta}}\left(\frac{p_{\perp}}{2}\right), \quad v_{2}^{B}\left(p_{\perp}\right) \approx v_{2}^{\alpha}\left(\frac{p_{\perp}}{3}\right)+v_{2}^{\beta}\left(\frac{p_{\perp}}{3}\right)+v_{2}^{\gamma}\left(\frac{p_{\perp}}{3}\right) .
$$

If all quark flavours carry the same elliptic flow, this simplifies further to [60]

$$
v_{2}^{h}\left(p_{\perp}\right) \approx n v_{2}^{q}\left(\frac{p_{\perp}}{n}\right) .
$$


In other words, if we divide $v_{2}$ for a hadron by the number $n$ of its valence quarks and plot it against the scaled transverse momentum $p_{\perp} / n$, we should obtain a universal function $v_{2}^{q}\left(\frac{p_{\perp}}{n}\right)$ describing the elliptic flow of the quarks just before hadronization.
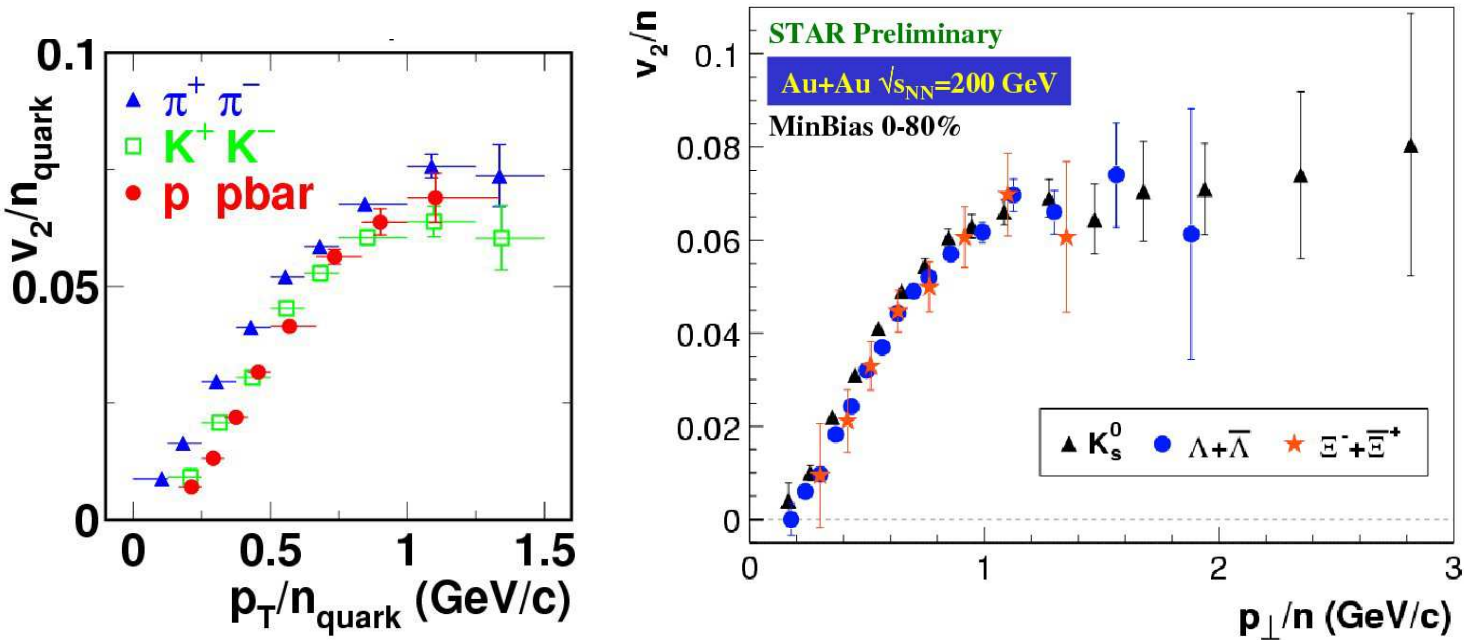

FIGURE 4. (color online) Elliptic flow per valence quark, $v_{2} / n$, as a function of transverse momentum per valence quark, $p_{\perp} / n$, for pions, kaons, protons, $\Lambda$ and $\Xi$ hyperons and their antiparticles. Data are from $200 \mathrm{~A} \mathrm{GeV} \mathrm{Au+Au} \mathrm{collisions} \mathrm{measured} \mathrm{by} \mathrm{PHENIX} \mathrm{[12]} \mathrm{(left} \mathrm{panel)} \mathrm{and} \mathrm{STAR} \mathrm{[49]} \mathrm{(right} \mathrm{panel).}$ Universality of this curve suggests that it represents the partonic elliptic flow $v_{2}^{\text {parton }}\left(p_{\perp}^{\text {parton }}\right)$, with no apparent difference between light and strange quark elliptic flow.

Figure 4 shows that this universal scaling works excellently. There is no indication for different elliptic flows for light and strange quarks. The common parton elliptic flow shows the same generic form as the individual hadronic curves, following the hydrodynmic rise at low $p_{\perp}$ up to about $p_{\perp}=750 \mathrm{MeV}$ where it breaks away from hydrodynamics, eventually saturating at a level of about $7 \%$ (for minimum bias $\mathrm{Au}+\mathrm{Au}$ collisions) above $p_{\perp}=1 \dot{\mathrm{GeV}}$. Note that, on the parton level, hydrodynamics works only out to about twice the mean transverse momentum; the coalescence mechanism ensures that this is sufficient for mesons and baryons to behave hydrodynamically to much larger $p_{\perp}$, namely about $1.5 \mathrm{GeV}$ for mesons and about $2.3 \mathrm{GeV}$ for baryons. Pentaquarks [63] should therefore show hydrodynamic behaviour up to $p_{\perp}=3.8 \mathrm{GeV} / c$, and their elliptic flow should saturate at $35 \%$ !

Another important remark is that the extraction of the partonic flow from the rescaled hadronic elliptic flows is only possible because hydrodynamics breaks down at high $p_{\perp}$. If the elliptic flow continued to follow the almost linear rise to higher $p_{\perp}$, rescaling both vertical and horizontal axes would simply reproduce this linear curve, and nothing could be learned. Since breakdown of hydrodynamics produces a structure (shoulder) in the function $v_{2}\left(p_{\perp}\right)$ and this shoulder moves under rescaling of the axes, the model can be tested and verified. The strongest evidence for the coalescence model comes from the fact that the shoulder of $v_{2}$ happens for different collision centralities at different absolute values for $v_{2}$ and at slightly different $p_{\perp}$ values; however, the quark number scaling of the shoulder position works at all impact parameters, i.e. different hadronic $v_{2}$-curves collapse onto a single partonic $v_{2}$ curve at each collision centrality [44].

Careful readers will note that for $p_{\perp}<750 \mathrm{MeV}$ the extracted partonic $v_{2}$ is not quite 
universal. In particular the differential partonic elliptic flow extracted from pions seems to be higher than that from the other hadrons (left panel in Figure 4). But this is only a reflection of the hydrodynamic mass splitting (remember: hydro works at low $p_{\perp}$ !) which, of course, cannot be scaled away by rescaling the axes. The coalescence model does not work at low $p_{\perp}$ (in particular, it violates entropy conservation because it reduces the number of entropy carriers by more than a factor 2 !); it can only be used at sufficiently high $p_{\perp}$ where coalescence is a relatively rare process [60].

Clearly, the coalescence model makes essential use of the concept of deconfined (uncorrelated) quarks with independent phase-space distributions. I do not see how the fact that it works so perfectly can be interpreted in any other way than to provide at least indirect evidence for quark deconfinement in the early partonic phase.

\section{Conclusions: Have we seen the QGP?}

As the astute reader of these pages must have already concluded from my repeated use of the acronym QGP for the dense early collision stage, my own answer to this question is: YES! The quantitative success of the hydrodynamic model in describing the bulk of particles emitted from $\mathrm{Au}+\mathrm{Au}$ collisions at RHIC provides compelling proof that thermalized matter at unprecedented energy densities $\langle e\rangle>100 e_{\mathrm{nm}}$ has been created. $\left(e_{\mathrm{nm}}=0.125 \mathrm{GeV} / \mathrm{fm}^{3}\right.$ is the energy density of cold nuclear matter inside atomic nuclei.) There is only one known viable theoretical concept that can describe thermalized matter at such energy densities: the Quark-Gluon Plasma. The mass splitting of the elliptic flow supports (but at the present accuracy does not prove) the hypothesis that this plasma is separated from normal hadronic matter by a phase transition (i.e. a soft region in the equation of state). The validity of the ideal fluid hydrodynamic model implies very severe constraints for the thermalization time scale and transport properties of this plasma: the required $\tau_{\mathrm{eq}}<1 \mathrm{fm} / c$ cannot be obtained with standard perturbative QCD scattering mechanisms and requires (presently unknown) non-perturbative physics which makes the QGP created at RHIC behave like a strongly interacting, almost ideal fluid rather than a weakly interacting parton gas.

One might worry that such non-perturbative processes might also modify the color deconfinement and chiral symmetry properties usually associated with the QGP, and transverse flow patterns do not, of course, provide any direct information about these characteristics. I showed that indirect arguments for color deconfinement can be extracted from the pattern in which the hydrodynamic description of hadron spectra begins to break down at intermediate transverse momenta: This pattern is consistently described by a quark-coalescence model, in which collective flow patterns are first imprinted on the momentum distributions of deconfined (uncorrelated) quarks in the early partonic phase and then transferred to the late hadronic phase by a quark recombination mechanism.

Another indirect argument going in the same direction is based on the success of the grand canonical thermodynamic ensemble in describing all the measured hadron abundance ratios [30]. This approach works extremely well even for multistrange hyperons and antihyperons, with a fully equilibrated strange particle sector (in contrast to elementary particle collisions and nuclear collisions at lower energies where strangeness is 
found to be suppressed relative to the grand canonical approach and requires taking into account, via canonical constraints and/or additional strangeness suppression factors, local correlations between strange quarks and antiquarks [64]). This implies that, say, in the production of an $\Omega$ hyperon, strangeness can be balanced by kaons or anti-hyperons anywhere in the fireball volume, including its far opposite edge. In other words, any memory of the fact that the microscopic strangeness production process in QCD is $l o$ cal, i.e. $s$ and $\bar{s}$ are created pairwise in a point, has been completely lost by the time strange hadrons form, and at hadronization the strange quarks and antiquarks are completely uncorrelated and equally likely to be found at any point in the fireball. It is very difficult, if not impossible, to explain this without invoking strong rescattering dynamics among deconfined quarks and gluons before the onset of hadron formation.

\section{Acknowledgements}

The author thanks the organizers of the school and workshop for their warm hospitality and for generating an intellectually very stimulating environment. This work was supported by the U.S. Department of Energy under contract DE-FG02-01ER41190.

\section{REFERENCES}

1. Ollitrault, J.-Y., Phys. Rev. D 46, 229 (1992).

2. Voloshin, S.A., and Zhang, Y., Z. Phys. C 70, 665 (1996).

3. Sorge, H., Phys. Rev. Lett. 78, 2309 (1997); ibid. 82, 2048 (1999).

4. Kolb, P. F., Sollfrank, J., and Heinz, U., Phys. Lett. B 459, 667 (1999); and Phys. Rev. C 62, 054909 (2000).

5. Karsch, F., Nucl. Phys. A698, 199c (2002).

6. Shuryak, E. V., Phys. Rev. C 61034905 (2000).

7. Li, B.-A., Phys. Rev. C 61021903 (R) (2000).

8. Zhang, B., Gyulassy, M., and Ko, C. M., Phys. Lett. B 455, 45 (1999).

9. Molnar, D., and Gyulassy, M., Nucl. Phys. A697, 495 (2002), Erratum ibid. A703, 893 (2002).

10. Voloshin, S. A., and Poskanzer, A. M., Phys. Lett. B 47427 (2000).

11. Ackermann, K. H., et al. (STAR Collab.), Phys. Rev. Lett. 86, 402 (2001); Adler, C., et al. (STAR Collab.), Phys. Rev. Lett. 87, 182301 (2001); ibid. 89, 132301 (2002); Phys. Rev. C 66, 034904 (2002); and Phys. Rev. Lett. 90, 032301 (2003).

12. Adcox, K., et al. (PHENIX Collab.), Phys. Rev. Lett. 89, 212301 (2002); Adler, S. S., et al. (PHENIX Collab.), Phys. Rev. Lett. 91, 182301 (2003).

13. Back, B. B., et al. (PHOBOS Collab.), Phys. Rev. Lett. 89, 222301 (2002); and arXiv:nucl-ex/0406021

14. Teaney, D., Lauret, J., and Shuryak, E. V., Phys. Rev. Lett. 86, 4783 (2001); and arXiv:nucl-th/0110037

15. Kolb, P. F., Huovinen, P., Heinz, U., and Heiselberg, H., Phys. Lett. B 500, 232 (2001).

16. Huovinen, P., Kolb, P. F., Heinz, U., Ruuskanen, P. V., and Voloshin, S. A., Phys. Lett. B 503, 58 (2001).

17. Kolb, P. F., Heinz, U., Huovinen, P., Eskola, K. J., and Tuominen, K., Nucl. Phys. A696, 175 (2001).

18. This notation was suggested by T. D. Lee, as quoted in Gyulassy, M., arXiv:nucl-th/0403032

19. Teaney, D., Phys. Rev. C 68, 034913 (2003), and private communication.

20. Rapp, R., Phys. Rev. C 66, 017901 (2002).

21. Hirano, T., and Tsuda, K., Phys. Rev. C 66, 054905 (2002).

22. Teaney, D., arXiv:nucl-th/0204023 
23. Rischke, D. H., "Fluid dynamics for relativistic nuclear collisions", in Hadrons in Dense Matter and Hadrosynthesis, edited by J. Cleymans et al., Springer Lecture Notes in Physics 516, Springer Verlag, Heidelberg, 1999, p.21 |nucl-th/9809044|.

24. Cooper, F., and Frye, G., Phys. Rev. D 10, 186 (1974).

25. Schnedermann, E., and Heinz, U., Phys. Rev. C 50, 1675 (1994).

26. Bass, S.A., and Dumitru, A., Phys. Rev. C 61, 064909 (2000).

27. Bjorken, J. D., Phys. Rev. D 27, 140 (1983).

28. Lee, K. S., Rhoades-Brown, M., and Heinz, U., Phys. Rev. C 37, 1452 (1988).

29. Sollfrank, J., et al., Phys. Rev. C 55, 392 (1997).

30. Braun-Munzinger, P., Magestro, D., Redlich, K., and Stachel, J., Phys. Lett. B 518, 41 (2001).

31. Kolb, P. F., and Rapp, R., Phys. Rev. C 67, 044903 (2003).

32. Sollfrank, J., Koch, P., and Heinz, U., Phys. Lett. B 252, 256 (1990); and Z. Phys. C 52, 593 (1991).

33. Lee, K. S., Heinz, U., and Schnedermann, E., Z. Phys. C 48, 525 (1990); Schnedermann, E., Sollfrank, J., and Heinz, U., Phys. Rev. C 48, 2462 (1993).

34. Heinz, U., and Kolb, P. F. Nucl. Phys. A702, 269 (2002).

35. Chujo, T., et al. (PHENIX Collab.), Nucl. Phys. A715, 151c (2003).

36. Barannikova, O., Wang, F., et al. (STAR Collab.), Nucl. Phys. A715, 458c (2003).

37. Wosiek, B., et al. (PHOBOS Collab.), Nucl. Phys. A715, 510c (2003).

38. Ouerdane, D., et al. (BRAHMS Collab.), Nucl. Phys. A715, 478c (2003).

39. Suire, C., et al. (STAR Collab.), Nucl. Phys. A715, 470c (2003).

40. Adams, J., et al. (STAR Collab.), Phys. Rev. Lett. 92, 182301 (2004).

41. Kolb, P. F., and Heinz, U., "Hydrodynamic description of ultrarelativistic heavy-ion collisions", in Quark-Gluon Plasma 3, edited by R. C. Hwa and X. N. Wang, World Scientific Publ. Co., Singapore, 2004, p.634 |arXiv:nucl-th/0305084|.

42. Heinz, U., and Kolb, P. F., "Two RHIC puzzles: Early thermalization and the HBT problem", in Proc. 18th Winter Workshop on Nuclear Dynamics, edited by R. Bellwied, J. Harris, and W. Bauer, EP Systema, Debrecen, Hungary, 2002, p.205 |arXiv:hep-ph/0204061|.

43. Adams, J., et al. (STAR Collab.), Phys. Rev. Lett. 92, 052302 (2004).

44. Sorensen, P. R., "Kaon and Lambda production at intermediate $p(T)$ : Insights into the hadronization of the bulk partonic matter created in $\mathrm{Au}+\mathrm{Au}$ collisions at RHIC", Ph.D. thesis, arXiv:nucl-ex/0309003

45. Hirano, T., Phys. Rev. C 65, 011901 (2002).

46. Heinz, U., and Kolb, P. F., J. Phys. G 30, S1229 (2004).

47. Alt, C., et al. (NA49 Collab.), Phys. Rev. C 68, 034903 (2003).

48. Agakichiev, G., et al. (CERES/NA45 Collab.), Phys. Rev. Lett. 92, 032301 (2004).

49. Castillo, J., et al. (STAR Collab.), arXiv:nucl-ex/0403027.

50. Bleicher, M., and Stöcker, H., Phys. Lett. B 526, 309 (2002).

51. Heinz, U., and Wong, S. M. H., Phys. Rev. C 66, 014907 (2002).

52. Muronga, A., contribution to this volume.

53. Schweda, K., et al. (STAR Collab.), arXiv:nucl-ex/0403032

54. Adler, S. S., et al. (PHENIX Collab.), Phys. Rev. Lett. 91, 172301 (2003).

55. Voloshin, S. A., Nucl. Phys. A715, 379 (2003).

56. Dover, C. B., Heinz, U., Schnedermann, E., and Zimányi, J., Phys. Rev. C 44, 1636 (1991).

57. Scheibl, R., and Heinz, U., Phys. Rev. C 59, 1585 (1999).

58. Greco, V., Ko, C. M., and Lévai, P., Phys. Rev. Lett. 90, 202302 (2003); and Phys. Rev. C 68, 034904 (2003).

59. Fries, R. J., Müller, B., Nonaka, C., and Bass, S. A., Phys. Rev. Lett. 90, 202303 (2003); and Phys. Rev. C 68, 044902 (2003).

60. Molnar, D., and Voloshin, S. A., Phys. Rev. Lett. 91, 092301 (2003).

61. Lin, Z. w., and Ko, C. M., Phys. Rev. Lett. 89, 202302 (2002).

62. Lin, Z. w., and Molnar, D., Phys. Rev. C 68, 044901 (2003).

63. Praszalowicz, M., in Skyrmions and Anomalies, edited by M. Jezabeck and M. Praszalowicz, World Scientific, 1987, p.112.

64. Braun-Munzinger, P., Redlich, K., and Stachel, J., "Particle production in heavy ion collisions", in Quark-Gluon Plasma 3, edited by R. C. Hwa and X. N. Wang, World Scientific Publ. Co., Singapore, 2004, p.491 |arXiv:nucl-th/0304013|. 